\title{
Focused issue on micro-/nano-robotics
}

\author{
Kenn Oldham ${ }^{1} \cdot$ Dong Sun ${ }^{2} \cdot$ Yu Sun ${ }^{3}$
}

Published online: 16 November 2018

(c) Springer Nature Singapore Pte Ltd. 2018

Micro-/nano-robotics research applies principles and knowledge from the broader robotics field to applications characterized by interaction with the external world at size scales of microns to nanometers. Micro- or nano-robotic systems may themselves be miniature in size, or they may be designed specifically to interact with micro- or nano-sized structures. The ability to interact in automated or semi-automated fashion with the environment at such scales generates unique new opportunities for scientific study, medical diagnosis and treatment, precision manufacturing, and exploration, among other applications. This focused section on micro-/ nano-robotics presents eight ongoing research activities in the miniature robotics area.

Arguably the most prominent motivation for micro-/nanorobotic research is to enable new interactions with biological organisms, whether for purposes of basic research or to generate new avenues for diagnosis and treatment of disease. "Passive-Active Hybrid Release Strategy for Micro-object Separation”, by Crimp, Suhaimi, and Avci, demonstrates a robotic system to rapidly pick-and-place microbeads, as a step toward oocyst manipulation for biomedical studies and precision medicine. This requires a close examination of adhesion force effects on micro-manipulator and cell/ object interaction, to ensure that objects can be held and then released by manipulators at desired locations.

A companion paper, "Auto-focusing Approach on Multiple Micro Objects using the Prewitt Operator", by Lofroth and Avci, is intended to further improve speed of micromanipulation tasks by increasing the efficiency with which

Kenn Oldham

oldham@umich.edu

1 Mechanical Engineering, University of Michigan, 2380C G.G. Brown Laboratory, 2350 Hayward St, Ann Arbor, MI 48109, USA

2 Biomedical Engineering, City University of Hong Kong, Room Y5-409, 5/F, Yellow Zone, Yeung Kin Man Academic Building, 83 Tat Chee Avenue, Kowloon Tong, Hong Kong

3 Mechanical Engineering, University of Toronto, MC 419, 5 King's College Road, Toronto, ON M5S 3G8, Canada micro objects may be identified and made the center of the focal plane when using optical imaging for feedback and tracking. This is especially important when the objects to be tracked are motile biological specimens that may move in and out of the focal plane.

Beyond physical contact or perturbation, optical and thermal manipulation techniques provide avenues for designing, altering, or assembling nano-scale systems. "Nano-assembly and Welding of Gold Nanorods based on DNA Origami and Plasmon-induced Laser Irradiation”, by Liu, Liu, and Shen, introduces a method for using DNA templates to induce rotation and positioning of gold nanorods to ensure that laser welding will bond the nanorods in desired orientations. The technique has potential to produce new material architectures and micro-robotic assemblies.

Yet another approach to manipulation and locomotion at the nano-scale is electromagnetic coupling, especially in a fluid, whether to propel nano-robots or sort and position micro objects. "Pairwise Controllability and Motion Primitives for Micro-Rotors in a Bounded Stokes Flow", by Buzhardt, Fedonyuk, and Tallapragada, examines the fundamental theoretical interaction between microswimmers modeled as rotlets in low Reynolds number flow, to show that combinations of multiple rotlets may produce motions that would be impossible for an individual, and that these motions are controllable in a bounded domain.

Small-scale manipulation can also be bridged between biomedical and nano-manufacturing and metrology applications, whenever circumstances requires extremely high accuracy (nanometer-level) precision in object positions. Real-time Motion Planning of Multiple Nanowires in Fluid Suspension under Electric-Field Actuation by Yi, Yu, and Shan addresses scalability of nano-manipulation procedures to rapid positioning of multiple nanostructures, using efficient search algorithms while dealing with many of the complex small-scale fluid-structure interactions encountered when manipulating biological specimens.

Nanometer level positioning is likewise a critical issue in metrology applications such as atomic force microscopy and transmission electron microscopy. A robotic platform 
or stage for performing sample positioning in such settings could improve flexibility, speed, and range for precision imaging and assembly. "A 3D-Printed 3-DOF Tripedal Micro robotic Platform for Unconstrained and Omnidirectional Sample Positioning", by Adibnazari, Nagel, and Leang, demonstrates that a low-cost, rapid-prototyped micro-robot for this setting can begin to harness these benefits while maintaining high-accuracy positioning.

Returning to the theme of biomedical applications, micropositioning apparatus have also been developed for use in medical procedures, and "A Large Air Gap Magnetic Levitator for Intra-Arterial Positioning of a Clinical Microcatheter: Modeling and Control", by Zaccardo, Miller, and Buckner, examines how electromagnetic levitation might be utilized for precise catheter insertion. Such catheters are used, for example, in delivery of radioactive microspheres for cancer targeting, and improved positioning may enhance efficacy and reduce unwanted tissue damage of cancer treatment.

Finally, research to produce fully autonomous microrobots also continues in a number of laboratories around the world. "Design and Analysis of Varied Gaits in Eleastic Vibratory Milli-Robots", uses a rapid-prototyped centimeter-scale testbed to explore one approach to dynamically expanding mobility of robots based on micro electromechanical system (MEMS) fabrication processes, in which motion is primarily dependent on elastic deformation of appendages and mechanisms.

Together, we believe the above articles provide a useful survey of developments in the field of micro- and nanorobotics, addressing issues of small-scale manipulation, measurement, planning, assembly, locomotion, and control. We thank the editor-in-chief and staff of the International Journal of Intelligent Robotics and Applications for this opportunity to focus on activity in the field, and hope that readers will find much value in this issue.

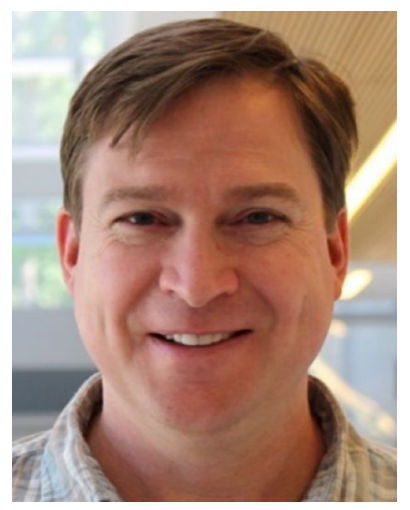

Kenn Oldham is an Associate Professor of Mechanical Engineering at the University of Michigan, Ann Arbor, USA. Prof. Oldham received the Ph.D. in Mechanical Engineering from the University of California at Berkeley and a B.S. in Mechanical Engineering from Carnegie Mellon University. He joined the University of Michigan following a post-doctoral fellowship at the U.S. Army Research Laboratory. Prof. Oldham and his research group study the intersection of control systems and micro-scale sensing and actuation, with interests in design for controllability, optimal and robust control, and novel sensor and actuator design. Applications of this research include terrestrial micro-robotics, endoscopic microscopy, and inertial and physiological sensing. Prof. Oldham is currently serving as Associate Chair for Undergraduate Education for Mechanical Engineering and as an Associate Director of the Michigan Center of Integrative Research in Critical Care at the University of Michigan.

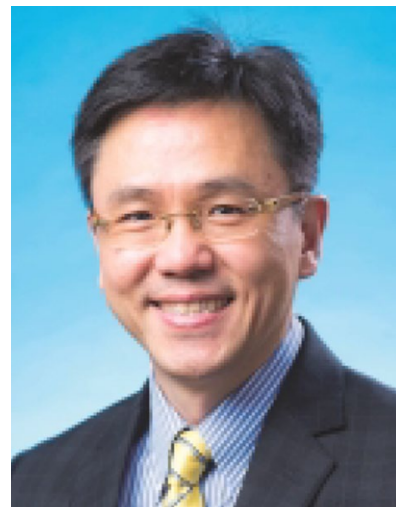

Sun Dong is the Chair Professor of Biomedical Engineering at the City University of Hong Kong. Prof. Sun received the $\mathrm{Ph} . \mathrm{D}$. in Robotics and Automation from the Chinese University of Hong Kong, an M.S. in Precision Instrument and Biomedical Engineering from Tsinghua University, Beijing, China, and a B.S. in Precision Instrument and Mechanology from Tsinghua University. Prof. Sun has extensive research activity in robotics and cell-based bioengineering, including automation of robotic cell handling and micro-surgery tasks and development of synchronization control for multirobot systems with applications to advanced manufacturing and micro-processing automation. He is a Fellow of IEEE, Fellow of HKIE, P.Eng. (Canada) and Member of ASME. His research has generated 380 technical papers, with multiple best paper awardees, 15 books and book chapters, and 10 patents.

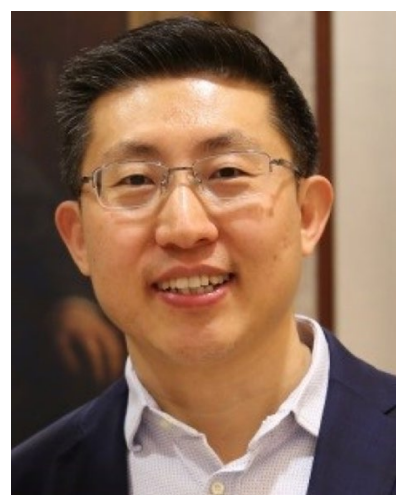

Yu Sun is a Professor of Mechanical Engineering at the University of Toronto, Ontario, Canada and Canada Research Chair in Micro and Nano Engineering Systems. $\mathrm{He}$ received the Ph.D. in Mechanical Engineering from the University of Minnesota, an Minneapolis, USA, an M.S. degree in Electrical Engineering from The University of Minnesota, an M.S. degree from the Institute of Automation, Chinese Academy of Automation, Beijing, China and a B.S. from Dalian University of Technology, Dalian, China. Prof. Sun's Advanced Micro and Nanosystems Lab designs and constructs enabling micro and nanosystems (e.g., micronano robotic systems and MEMS/microfluidic devices) for automated operation at the micro and nanometer scales, with applications to fundamental studies and clinical/industrial applications. Prof. Sun and is a McLean Senior Faculty Fellow and holds joint appointments in the Institute of Biomaterials and Biomedical Engineering and Department of Electrical and Computer Engineering at the University of Toronto. $\mathrm{He}$ is the Faculty Director of the University Nanofabrication Centre and a member of the Canadian Academy of Engineering. He has received multiple honors and best paper awards in the fields of robotics and micro-/nano-systems, and is a Fellow of IEEE, ASME, the Engineering Institute of Canada, and the American Association for Advancement of Science. 